\title{
Heavy Ethanol Intoxication Increases Proinflammatory Cytokines and Aggravates Hemorrhagic Shock-Induced Organ Damage in Rats
}

\author{
Tsung-Ming Hu, ${ }^{1,2}$ Ru-Ping Lee, ${ }^{3}$ Chung-Jen Lee, ${ }^{4}$ Yi-Maun Subeq, ${ }^{3}$ \\ Nien-Tsung Lin, ${ }^{5}$ and Bang-Gee Hsu ${ }^{6,7}$ \\ ${ }^{1}$ Institute of Medical Sciences, Tzu Chi University, Hualien, Taiwan \\ ${ }^{2}$ Department of Psychiatry, Yuli Veterans Hospital, Hualien, Taiwan \\ ${ }^{3}$ Department of Nursing, Tzu Chi University, Hualien, Taiwan \\ ${ }^{4}$ Department of Nursing, Tzu Chi College of Technology, Hualien, Taiwan \\ ${ }^{5}$ Institute of Microbiology, Immunology and Molecular Medicine, Tzu Chi University, Hualien, Taiwan \\ ${ }^{6}$ Department of Nephrology, Tzu Chi General Hospital, Hualien, Taiwan \\ ${ }^{7}$ School of Medicine, Tzu Chi University, Hualien, Taiwan
}

Correspondence should be addressed to Bang-Gee Hsu; gee.lily@msa.hinet.net

Received 15 April 2013; Revised 2 July 2013; Accepted 13 August 2013

Academic Editor: Borna Relja

Copyright (C) 2013 Tsung-Ming Hu et al. This is an open access article distributed under the Creative Commons Attribution License, which permits unrestricted use, distribution, and reproduction in any medium, provided the original work is properly cited.

Hemorrhagic shock (HS) following acute alcohol intoxication can increase proinflammatory cytokine production and induce marked immunosuppression. We investigated the effects of ethanol on physiopathology and cytokine levels following HS in acutely alcohol-intoxicated rats. Rats received an intravenous injection of $5 \mathrm{~g} / \mathrm{kg}$ ethanol over $3 \mathrm{~h}$ followed by HS induced by withdrawal of $40 \%$ of total blood volume from a femoral arterial catheter over 30 min. Mean arterial pressure (MAP) and heart rate (HR) were monitored continuously for $48 \mathrm{~h}$ after the start of blood withdrawal. Biochemical parameters, including hemoglobin, ethanol, glutamic oxaloacetic transaminase (GOT), glutamic pyruvic transaminase (GPT), blood urea nitrogen (BUN), creatinine (Cre), lactic dehydrogenase (LDH), and creatine phosphokinase (CPK), were measured at 30 min before induction of HS and $0,1,3,6,9$, $12,18,24$, and $48 \mathrm{~h}$ after HS. Serum tumor necrosis factor- $\alpha$ (TNF- $\alpha$ ) and interleukin-6 (IL-6) levels were measured at 1 and $12 \mathrm{~h}$ after HS. The liver, kidneys, and lungs were removed for pathology at $48 \mathrm{~h}$ later. HS significantly increased HR, blood GOT, GPT, BUN, Cre, $\mathrm{LDH}, \mathrm{CPK}, \mathrm{TNF}-\alpha$, and IL-6 levels and decreased hemoglobin and MAP in rats. Acute ethanol intoxication further increased serum levels of GOT, GPT, BUN, Cre, LDH, CPK, TNF- $\alpha$ and IL- 6 elevation following HS. Acutely intoxicated rats exacerbated the histopathologic changes in the liver, kidneys, and lungs following HS.

\section{Introduction}

Traumatic injury is a leading cause of death and disability worldwide, and hemorrhagic shock (HS) is responsible for up to $40 \%$ of trauma deaths [1]. HS can lead to hemodynamic instability, decrease in oxygen delivery, and induce tissue hypoperfusion, leading to cellular hypoxia, organ damage, and death $[2,3]$. After HS, nuclear factor- $\kappa \mathrm{B}(\mathrm{NF}-\kappa \mathrm{B})$ is activated causing the expression of several proinflammatory cytokines, such as tumor necrosis factor- $\alpha$ (TNF- $\alpha$ ) or interleukin-6 (IL-6), and this series of events can, in turn, lead to multiple organ dysfunction [2-5].

Acute alcohol intoxication is a significant risk factor for traumatic injury and causes higher morbidity or mortality rates in patients with HS $[6,7]$. Acute alcohol intoxication is a clinically harmful condition that commonly follows the ingestion of a large amount of alcohol [8]. Not only risk of injury is increased by alcohol use, acute intoxication negatively affects severity of trauma-related immune compromise and recovery from trauma-related hospitalization [9]. 
Following HS, but also acute alcohol intoxication increases proinflammatory cytokine production and induces marked immunosuppression [6]. Hospital emergency rooms regularly see patients with HS as a result of accidents occurring while in a state of acute alcohol intoxication. This study used a rat model to explore how acute alcohol intoxication affects recovery from HS on cytokines (TNF- $\alpha$, and IL-6) and damage to organs (liver, kidney, and lung).

\section{Materials and Methods}

2.1. Preparation of Animals. Thirty-two male Wistar-Kyoto rats weighing 260-300 grams were purchased from the National Animal Center (Taipei, Taiwan). They were housed in the university Animal Center in a controlled environment at a temperature of $22 \pm 1^{\circ} \mathrm{C}$ with a 12-hour light/dark cycle. Food and water were provided ad libitum. The Animal Care and Use Committee of Tzu Chi University approved the experimental protocol.

The animals were anesthetized with ether inhalation for about $15 \mathrm{~min}$. During the period of anesthesia, a polyethylene catheter (PE-50) was inserted into the femoral artery to collect blood samples and was connected to a pressure transducer (Gould Instruments, Cleveland, OH, USA) to record arterial pressure $(\mathrm{AP})$ and heart rate $(\mathrm{HR})$ on a polygraph recorder (Power Lab, AD Instruments, Mountain View, CA, USA). Another PE-50 catheter was inserted into the femoral vein for intravenous administration of drugs or fluid. The operation was completed within $15 \mathrm{~min}$, leaving a small wound (less than $0.5 \mathrm{~cm}^{2}$ ). After the operation, the animals were placed in a conscious rat metabolic cage (Shingshieying Instruments, Hualien, Taiwan). Rats awoke soon after the operation, and acute alcohol intoxication was induced $24 \mathrm{~h}$ later, with the rats in a conscious state [10-12].

2.2. Acute Alcohol Intoxication. After PE-50 catheters were inserted into the femoral artery and femoral vein in rats $24 \mathrm{~h}$ later. Acute alcohol intoxication in rats was given $5 \mathrm{~g} / \mathrm{kg}$ ethanol in normal saline mixed to $4 \mathrm{~mL}$ intravenously over $3 \mathrm{~h}[13]$.

2.3. Hemorrhagic Shock. HS was induced by drawing blood from the femoral arterial catheter into a $10 \mathrm{~mL}$ syringe after acute alcohol intoxication. An infusion pump controlled the withdrawal rate to mimic a typical bleeding event. The amount withdrawn was $40 \%$ of total blood volume $(6 \mathrm{~mL} / 100 \mathrm{gm} \mathrm{BW}+0.77 \mathrm{~mL})$ over a period of $30 \mathrm{~min}$ [14]. The HS procedure was followed by resuscitation with $0.5 \mathrm{~mL}$ normal saline at $0,1,3,6,9,12,18,24$, and $48 \mathrm{~h}$ after HS. After blood withdrawal, the animals were continuously observed for $48 \mathrm{~h}$ and sacrificed later for pathological study [10-12].

2.4. Experimental Design. Animals were randomly divided into four groups. Rats in the Ethanol group $(n=8)$, were given $5 \mathrm{~g} / \mathrm{kg}$ ethanol in normal saline mixed to $4 \mathrm{~mL}$ intravenously over $3 \mathrm{~h}$ and were not subjected to HS [14]. Rats in the HS group $(n=8)$ received an intravenous drip of $4 \mathrm{~mL}$ normal saline for $3 \mathrm{~h}$ followed by induction of HS.
The Ethanol + HS group $(n=8)$ were given $5 \mathrm{~g} / \mathrm{kg}$ ethanol in normal saline mixed to $4 \mathrm{~mL}$ intravenously over $3 \mathrm{~h}$ after which HS was immediately induced. In the Vehicle group $(n=8)$, rats received an intravenous drip of $4 \mathrm{~mL}$ normal saline for $3 \mathrm{~h}$ and were not subjected to HS (Figure 1).

2.5. Blood Sample Analysis. Arterial blood samples were obtained to determine baseline values before heparinization. Heparin (2 IU/gm BW) in $1 \mathrm{~mL}$ normal saline was injected via the catheter into rats over $20 \mathrm{~min}$ [10-12]. Arterial blood samples $(0.5 \mathrm{~mL})$ were collected for measurement of glutamic oxaloacetic transaminase (GOT), glutamic pyruvic transaminase (GPT), blood urea nitrogen (BUN), creatinine (Cre), lactic dehydrogenase (LDH), creatine phosphokinase (CPK), and ethanol at $3 \mathrm{~h}$ before induction of $\mathrm{HS}$, and at $0,1,3,6,9,12$, 18,24 , and $48 \mathrm{~h}$ following $\mathrm{HS}$, while an equal volume of $0.5 \mathrm{~mL}$ normal saline was used for fluid resuscitation. Blood samples of about $0.1 \mathrm{~mL}$ for hemoglobin (Sysmex K-1000, Sysmex American, Mundelein, IL, USA) and of $0.4 \mathrm{~mL}$ blood samples were immediately centrifuged at $3,000 \mathrm{~g}$ for $10 \mathrm{~min}$. The serum was decanted and separated into two parts; one part was stored at $4^{\circ} \mathrm{C}$ within $1 \mathrm{~h}$ after collection for biochemical analysis. We measured serum levels of GOT, GPT, BUN, Cre, LDH, CPK, and ethanol with an autoanalyzer (COBAS C111, Roche Diagnostics, Basel, Switzerland) to obtain various biochemical data. The other part of the serum collected at $1 \mathrm{~h}$ after $\mathrm{HS}$ was stored at $-80^{\circ} \mathrm{C}$ for later measurement of TNF- $\alpha$ and IL- 6 concentrations [10-12].

2.6. TNF- $\alpha$ and IL-6 Measurement by ELISA. TNF- $\alpha$ and IL-6 concentrations in the blood samples were measured separately 1 and 12 hours after induction of HS by antibody enzyme-linked immunosorbent assay (ELISA) using commercial antibody pairs, recombinant standards, and a biotin-streptavidin-peroxidase detection system (Endogen, Rockford, IL, USA) as previously described [10-12]. Blood samples were collected in serum separator tubes. All reagents, samples, and working standards were brought to room temperature and prepared according to the manufacturer's directions. Reactions were quantified by optical density using an automated ELISA reader (Sunrise, Tecan Co., Grödingen, Austria) at 450/540 $\mathrm{nm}$ wavelengths.

2.7. Histological Examination. Rats were sacrificed 48 hours after induction of HS and the livers, kidneys, and lungs were immediately removed. Livers, kidneys, and lungs tissues specimens were fixed overnight in $4 \%$ buffered formaldehyde, processed using standard methods and stained with hematoxylin and eosin ( $\mathrm{H} \& \mathrm{E})$. One observer who was blinded to the group assignment performed the tissue analysis. The severity of liver injury observed in the tissue sections was scored as follows: 0 , no evidence or minimal evidence of injury; 1, mild injury consisting of cytoplasmic vacuolation and focal nuclear pyknosis; 2, moderate to severe injury with extensive nuclear pyknosis, cytoplasmic hypereosinophilia, and loss of intercellular borders; and 3, severe necrosis with disintegration of the hepatic cords, hemorrhage, and neutrophil infiltration [10-12]. The severity of renal tubular 


\begin{tabular}{|c|c|c|c|c|c|}
\hline \multirow{2}{*}{$\begin{array}{l}\text { Anesthesia } \\
\text { Insert catheter }\end{array}$} & \multirow[t]{2}{*}{ Rest } & Alcohol intoxication & Bleeding & \multirow[t]{2}{*}{ Observation } & \multirow[t]{2}{*}{ Sacrificed } \\
\hline & & No alcohol intoxication & No bleeding & & \\
\hline \multirow{8}{*}{$\begin{array}{l}32 \text { male } \\
\text { WKY rats }\end{array}$} & \multirow[b]{4}{*}{ Random } & \multirow[t]{2}{*}{ Ethanol $(n=8)$} & No HS & & \multirow{8}{*}{ Pathology } \\
\hline & & & \multirow[b]{2}{*}{ HS } & & \\
\hline & & \multirow{2}{*}{ Ethanol + HS $(n=8)$} & & & \\
\hline & & & 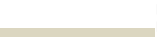 & & \\
\hline & & \multirow{2}{*}{ Vehicle $(n=8)$} & No HS & & \\
\hline & & & & & \\
\hline & & HS $(n=8)$ & HS & & \\
\hline & & & & & \\
\hline & $24 \mathrm{hr}$ & $3 \mathrm{hr}$ & 30 mins & $48 \mathrm{hr}$ & \\
\hline & & Pre & 0 & $\begin{array}{lllllll}1 & 3 & 6 & 9 & 12 & 18 & 24\end{array}$ & Bloc \\
\hline
\end{tabular}

FIGURE 1: Timeline of hemorrhage and blood sampling protocols for this experiment.

injury was scored by estimating the percentage of tubules in the cortex or the outer medulla that showed epithelial necrosis or had luminal necrotic debris, tubular dilation, and hemorrhage: 0 , none; $1,<5 \% ; 2,5$ to $25 \% ; 3,25$ to $75 \%$; and $4,>75 \%$ [10-12]. Lung injury was scored as follows: 0 , no evidence; 1 , mild injury; 2 , moderate injury; 3 , severe injury with lung edema, interstitial inflammatory cell infiltration, and hemorrhage [10-12]. All evaluations were made on five fields per section and five sections per organ.

2.8. Statistical Analysis. Data were expressed as mean \pm SD. Statistical comparisons between different groups at corresponding time points were made by repeated measures of two-way ANOVA followed by a post hoc test (Bonferroni's method). Histological scores were analyzed by the KruskalWallis test followed by the Dunn's test. A $P$ value less than 0.05 was considered statistically significant.

\section{Results}

3.1. Mean Arterial Pressure (MAP) and Heart Rate (HR). All rats were alive during the first $48 \mathrm{~h}$ of the study. The rats' mean arterial pressure (MAP) decreased rapidly after withdrawal of $40 \%$ of total blood volume from the femoral arterial catheter. MAP stayed relatively low during the $48 \mathrm{~h}$ after induction of HS (Figure 2(a)). Compared with the Vehicle group which was not subjected to HS, the Ethanol $+\mathrm{HS}$ group had decreased MAP at $0,1,3,6,9,12,18,24$, and $48 \mathrm{~h}$ after $\mathrm{HS}\left({ }^{*} \mathrm{P}<0.05\right.$; Figure $\left.2(\mathrm{a})\right)$. MAP was not significantly different between the Ethanol group and the Vehicle group (Figure 2(a)). Moreover, no significant difference was observed in MAP after HS when comparing the HS group with the Ethanol + HS group (Figure 2(a)). Heart rate (HR) was significantly increased during HS (Figure 2(b)). The HS group had increased tachycardia at $6,9,12,18,24$, and $48 \mathrm{~h}$ after HS compared with the Vehicle group $\left({ }^{*} P<0.05\right.$; Figure 2(b)). No significant difference in HR was observed after HS when we compared the HS group to the Ethanol + HS group (Figure 2(b)).

3.2. Serum Ethanol Level and Hemoglobin. Serum ethanol levels were significantly elevated after ethanol intravenous drip in the Ethanol group and the Ethanol + HS group, with the peak at $0 \mathrm{~h}$, then gradually decreasing to normal at $24 \mathrm{~h}$ (Figure 2(c)). Compared with the Vehicle group, the Ethanol group had higher serum ethanol levels at $0,1,3,6,9,12,18$, and $24 \mathrm{~h}$ after HS $\left({ }^{*} \mathrm{P}<0.05\right.$; Figure $\left.2(\mathrm{c})\right)$. HS did not affect serum ethanol levels when we compared the Ethanol only group with the Ethanol + HS group (Figure 2(c)). Hemoglobin gradually decreased after induction of HS (Figure $2(\mathrm{~d})$ ). Compared with the Vehicle group, the HS group showed decreased hemoglobin at 1, 3, 6, 9, 12, 18, 24, and $48 \mathrm{~h}$ after HS $\left({ }^{*} P<0.05\right.$; Figure $\left.2(\mathrm{~d})\right)$, but compared with the Vehicle group, the Ethanol group had increased hemoglobin at 0 , 1,3 , and $6 \mathrm{~h}$ after $\mathrm{HS}\left({ }^{+} P<0.05\right.$; Figure $\left.2(\mathrm{~d})\right)$. Compared with the HS only group, the Ethanol + HS group showed increased hemoglobin at $0 \mathrm{~h}$ and decreased hemoglobin at 18, 24 , and $48 \mathrm{~h}$ after HS ( ${ }^{\#} P<0.05$; Figure $2(\mathrm{~d})$ ). After ethanol intoxication following HS, hemoglobin increased first and then decreased compared with the HS only group.

\subsection{Glutamic Oxaloacetic Transaminase (GOT) and Glutamic} Pyruvic Transaminase (GPT). GOT and GPT are measurements of liver function. GOT gradually increased at 18,24 , and $48 \mathrm{~h}$ after induction of HS $\left({ }^{*} \mathrm{P}<0.05\right.$; Figure $\left.3(\mathrm{a})\right)$. Compared with the Vehicle group, the Ethanol group showed higher GOT levels at 18, 24, and $48 \mathrm{~h}$ after $\mathrm{HS}\left({ }^{+} P<0.05\right.$; Figure 3(a)). Compared with the HS only group, the Ethanol + HS group had higher levels of GOT at 1, 3, 9, 12, 18, 24, and 48 h $\left({ }^{\#} P<0.05\right.$; Figure $\left.3(\mathrm{a})\right)$. We observed no statistically significant difference in serum GOT in the Ethanol group compared with the HS group (Figure 3(a)). GPT gradually increased after induction of HS $\left({ }^{*} P<0.05\right.$; Figure 3(b)). Compared with the Vehicle group, the Ethanol only group 


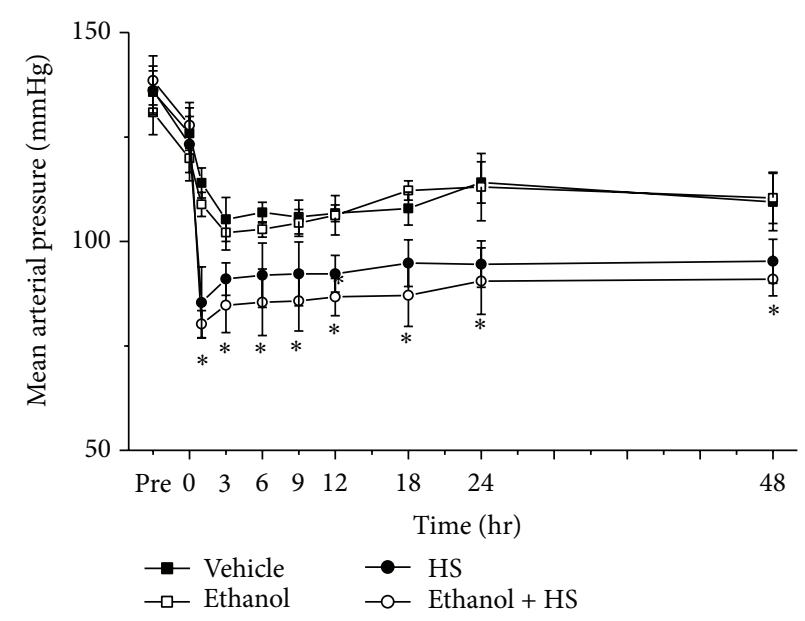

(a)

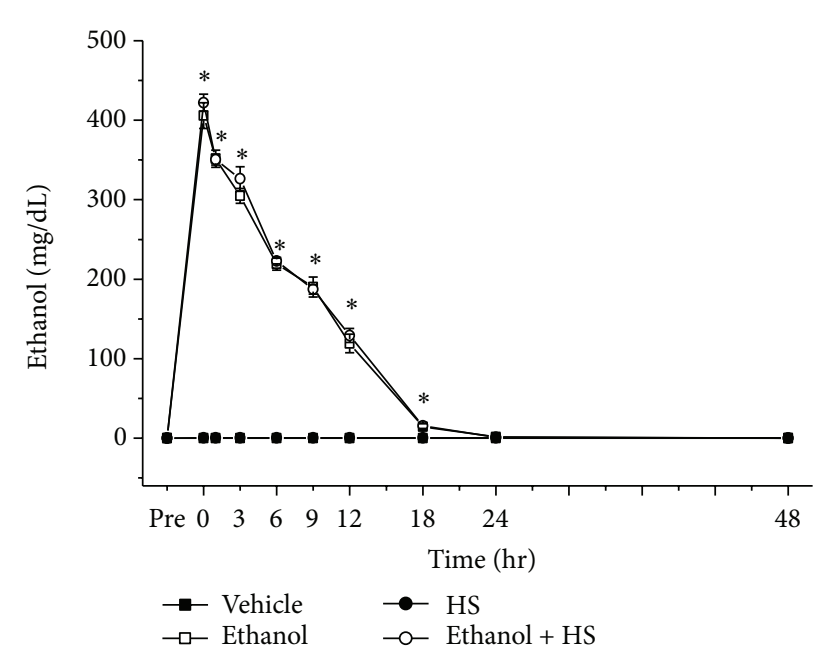

(c)

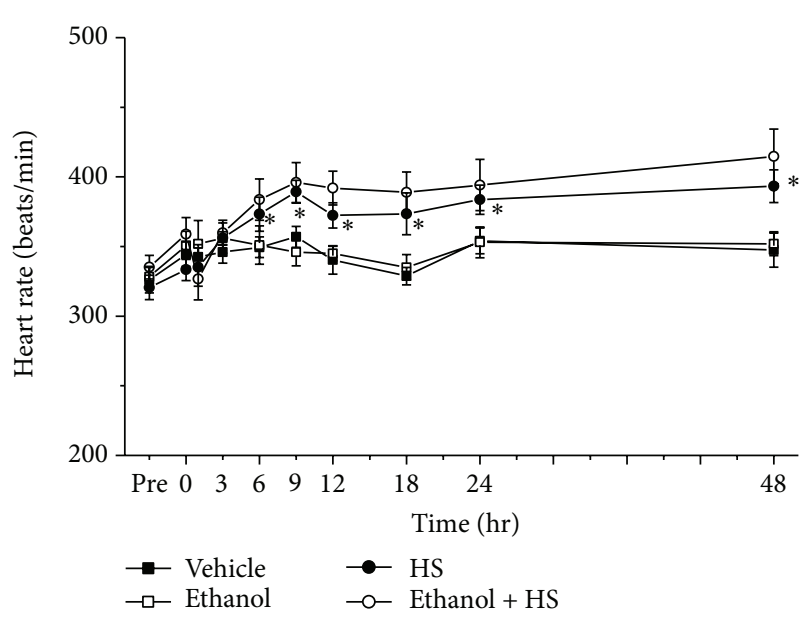

(b)

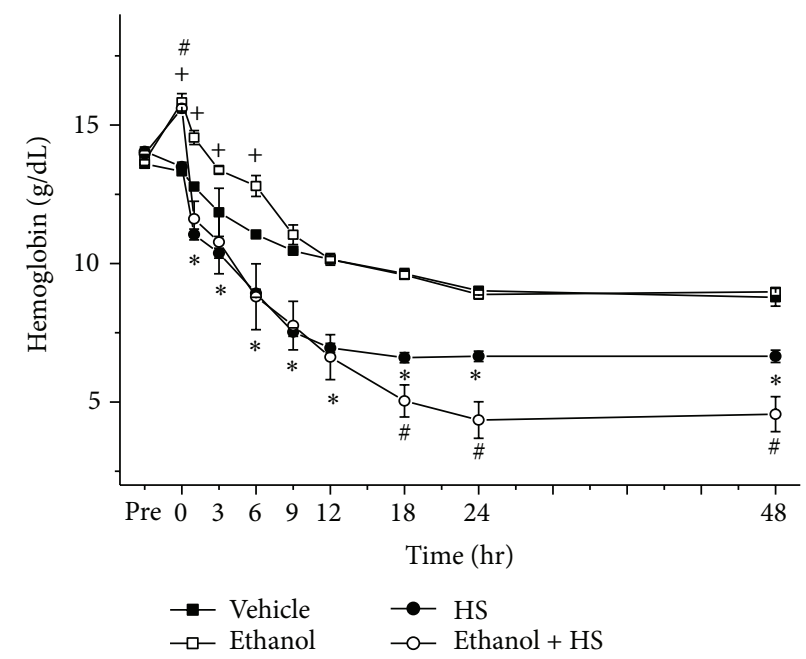

(d)

FIguRE 2: Changes in (a) mean arterial pressure, (b) heart rate, (c) serum ethanol concentration, and (d) hemoglobin following hemorrhagic shock in rats. ${ }^{*} P<0.05$ for the HS group compared with the Vehicle group. ${ }^{+} P<0.05$ for the Ethanol group compared with the Vehicle group. ${ }^{\#} P<0.05$ for the Ethanol + HS group compared with the HS group.

had increased GPT at $48 \mathrm{~h}$ after HS $\left({ }^{+} P<0.05\right.$; Figure 3(b)). Compared with the HS group, the Ethanol + HS group had even higher GPT at 3 and $48 \mathrm{~h}\left({ }^{\#} P<0.05\right.$; Figure 3(b)).

\subsection{Blood Urea Nitrogen (BUN), and Creatinine (Cre). BUN} and Cre are measured of kidney function. HS increased blood BUN at $1,3,6,9,18,24$, and $48 \mathrm{~h}\left({ }^{*} P<0.05\right.$; Figure 4(a)). Compared with the Vehicle group, the Ethanol group had increased BUN at $1,3,6$, and $9 \mathrm{~h}$ after $\mathrm{HS}\left({ }^{+} P<0.05\right.$; Figure 4(a)). Compared with the HS group, the Ethanol + HS group had even higher BUN at 1, 3, 6, 9, 12, 18, and $48 \mathrm{~h}$ ( ${ }^{\#} P<0.05$; Figure $4(\mathrm{a})$ ). No statistically significant difference was observed in serum BUN when comparing the Ethanol group with the HS group (Figure 4(a)). Serum Cre increased rapidly after induction of $\mathrm{HS}$. The serum Cre values increased at $1,3,6,9,12$, and $48 \mathrm{~h}$ after HS compared with the Vehicle group $\left({ }^{*} P<0.05\right.$; Figure $\left.4(\mathrm{~b})\right)$. But compared with the HS group, the Ethanol + HS group had even higher Cre at 1, 3, 6, $12,18,24$, and $48 \mathrm{~h}\left({ }^{\#} P<0.05\right.$; Figure $\left.4(\mathrm{~b})\right)$.
3.5. Lactic Dehydrogenase (LDH) and Creatine Phosphokinase $(C P K)$. The Ethanol group had increased LDH at 0 and $1 \mathrm{~h}$ compared with the Vehicle group $\left({ }^{+} P<0.05\right.$; Figure $\left.5(\mathrm{a})\right)$. The Ethanol + HS group had increased LDH at $0,1,18,24$, and $48 \mathrm{~h}$ compared with the HS group $\left({ }^{\#} P<0.05\right.$; Figure $\left.5(\mathrm{a})\right)$. HS increased blood CPK at $3,6,9,12$, and $18 \mathrm{~h}\left({ }^{*} P<0.05\right.$; Figure 5(b)). Compared with the Vehicle group, the Ethanol group showed increased $\mathrm{CPK}$ at 0 and $1 \mathrm{~h}\left({ }^{+} P<0.05\right.$; Figure 5(b)). Compared with the HS group, the Ethanol + HS group had still further increased CPK at $0,1,3,6,9,12,18,24$, and $48 \mathrm{~h}\left({ }^{\#} P<0.05\right.$; Figure 5(b)).

3.6. Tumor Necrosis Factor- $\alpha$ (TNF- $\alpha$ ) and Interleukin-6 (IL$6)$. HS greatly elevated serum TNF- $\alpha$ compared with the Vehicle group at 1 and $12 \mathrm{~h}\left({ }^{*} \mathrm{P}<0.05\right.$; Figure 6(a)). Prior administration of ethanol significantly increased the serum TNF- $\alpha$ at 1 and $12 \mathrm{~h}$ after induction of HS $\left({ }^{\#} P<0.05\right.$; Figure 6(a)). HS increased serum IL-6, compared with the Vehicle group at 1 and $12 \mathrm{~h}$ after induction of $\mathrm{HS}\left({ }^{*} \mathrm{P}<0.05\right.$; 


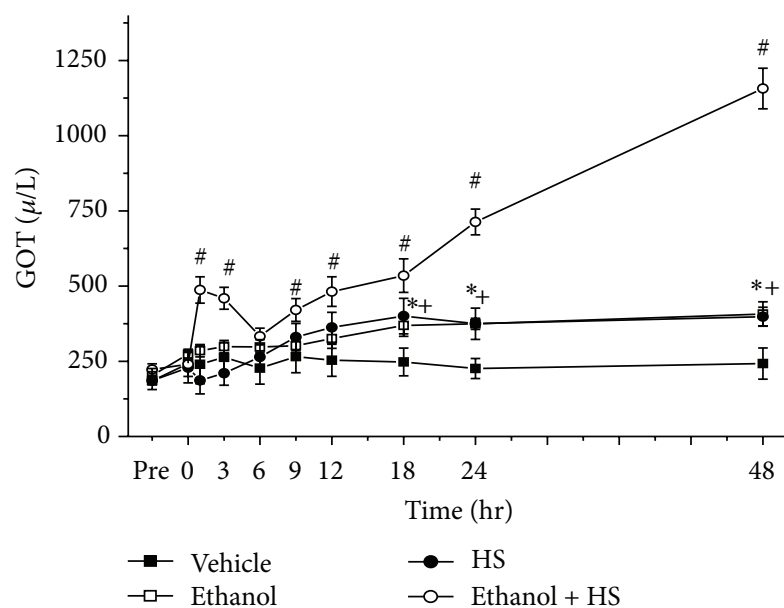

(a)

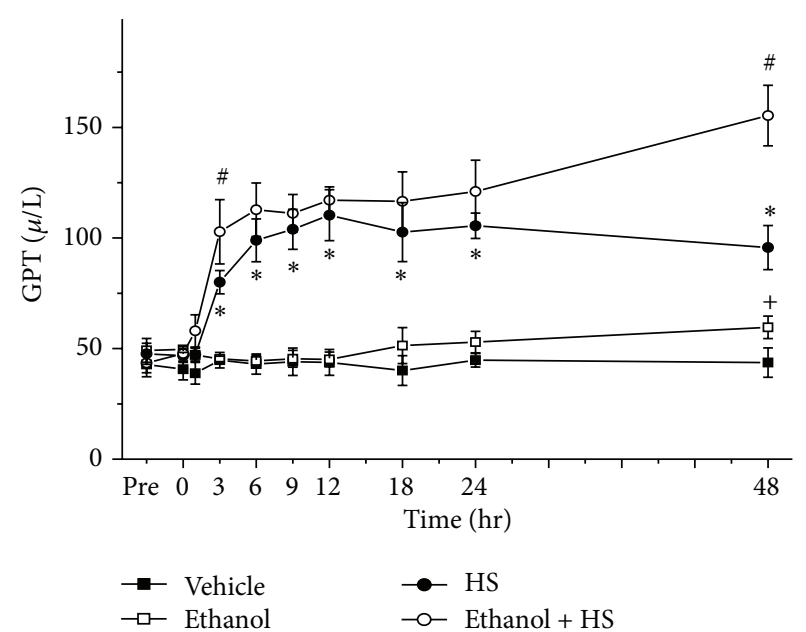

(b)

FIGURE 3: Changes in serum (a) glutamic oxaloacetic transaminase (GOT) and (b) glutamic pyruvic transaminase (GPT) after hemorrhagic shock in rats. ${ }^{*} P<0.05$ for the HS group compared with the Vehicle group. ${ }^{+} P<0.05$ for the Ethanol group compared with the Vehicle group. ${ }^{*} P<0.05$ for the Ethanol + HS group compared with the HS group.

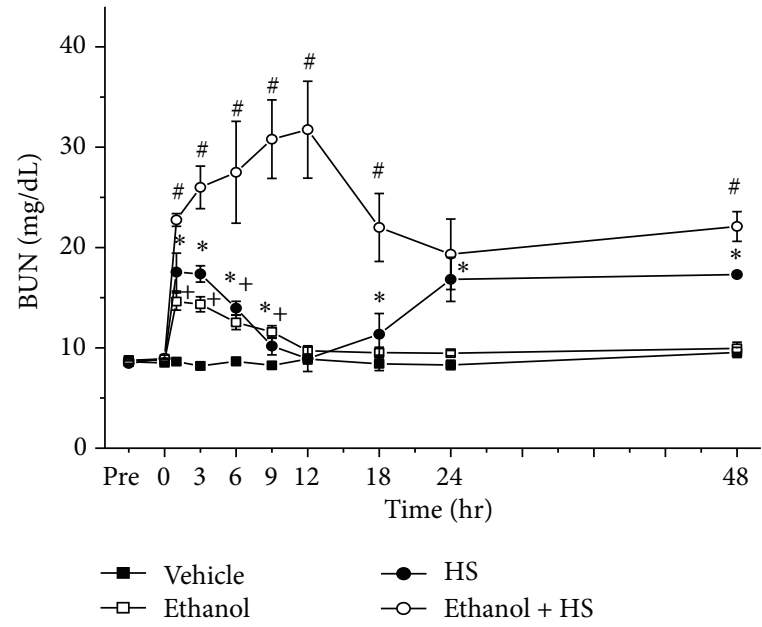

(a)

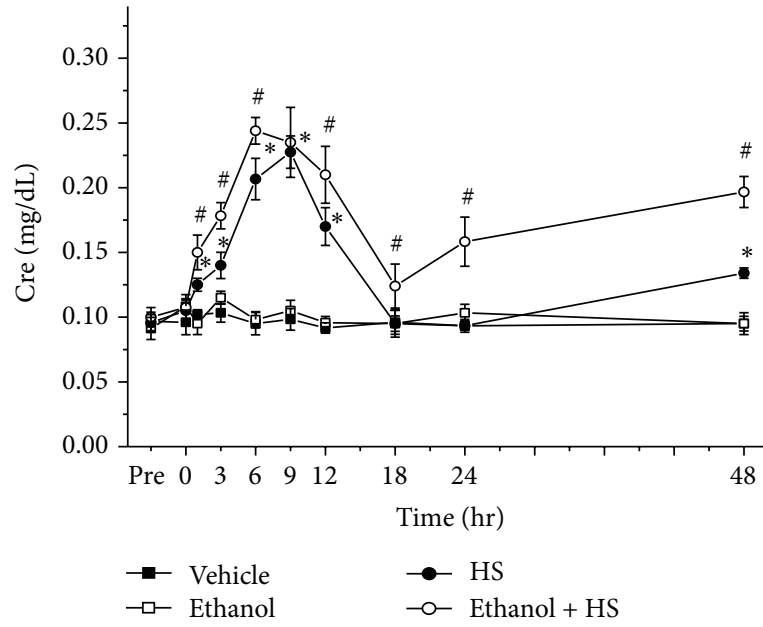

(b)

FIGURE 4: Changes in serum (a) blood urea nitrogen (BUN) and (b) creatinine (Cre) after hemorrhagic shock in rats. ${ }^{*} P<0.05$ for the HS group compared with the Vehicle group. ${ }^{+} P<0.05$ for the Ethanol group compared with the Vehicle group. ${ }^{\#} P<0.05$ for the Ethanol $+\mathrm{HS}$ group compared with the HS group.

Figure 6(b)). Compared with the HS only group, the Ethanol + HS group had even higher IL- 6 at 1 and $12 \mathrm{~h}$ after induction of HS ( ${ }^{\#} P<0.05$; Figure 6(b)).

3.7. Histopathology of Liver, Kidney, and Lung. Histopathologic analysis of $\mathrm{H} \&$ E-stained tissue sections from the liver, kidneys, and lungs after HS revealed hepatocyte necrosis and leukocytes infiltration in the liver (Figure 7(g)), tubular cell swelling, nuclear loss, tubular dilatation, and brush border loss in the kidney (Figure 7(h)). Pulmonary edema, hemorrhage, and interstitial polymorphonuclear (PMN) inflammatory cells infiltration in the lung were observed after HS (Figure 7(i)). Compared with the HS group, the Ethanol + HS group had greater histopathologic changes and hemorrhage in the liver, kidney, and lung (Figures $7(\mathrm{j}), 7(\mathrm{k})$, and $7(\mathrm{l})$ ). Compared with the HS group, the Ethanol + HS group had increased injury scores of the liver, kidney, and lung $\left({ }^{\#} P<\right.$ 0.05 ; Figures $7(\mathrm{~m}), 7(\mathrm{n})$, and $7(\mathrm{o}))$.

\section{Discussion}

This study found that intravenous heavy ethanol increased serum TNF- $\alpha$ and IL- 6 levels after HS and aggravated HS-induced organ damage (liver, kidney, and lung) in rats.

The prevalence of alcohol-related visits to U.S. trauma centers ranged from $26.2 \%$ to $62.5 \%$ [15]. Symptoms are 


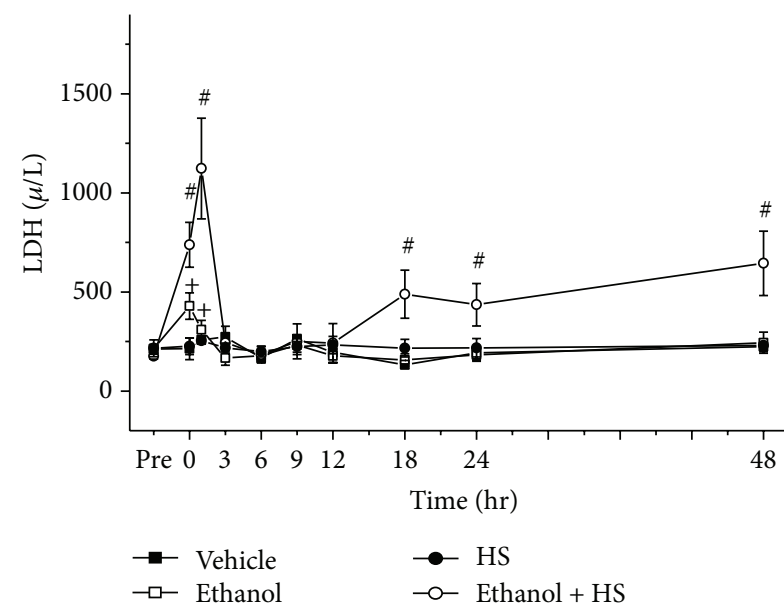

(a)

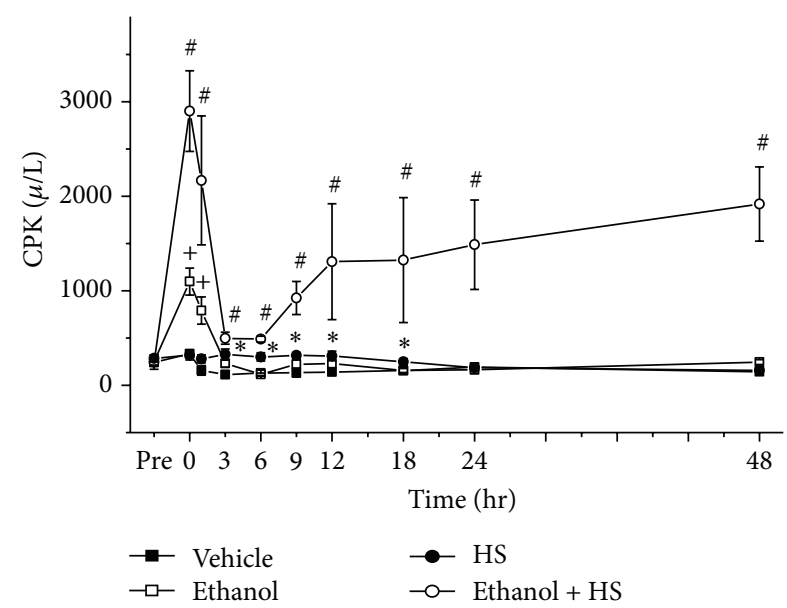

(b)

FIGURE 5: Changes in serum (a) lactic dehydrogenase (LDH), and (b) creatine phosphokinase (CPK) after hemorrhagic shock in rats. ${ }^{*} P<$ 0.05 for the HS group compared with the Vehicle group. ${ }^{+} P<0.05$ for the Ethanol group compared with the Vehicle group. ${ }^{*} P<0.05$ for the Ethanol + HS group compared with the HS group.

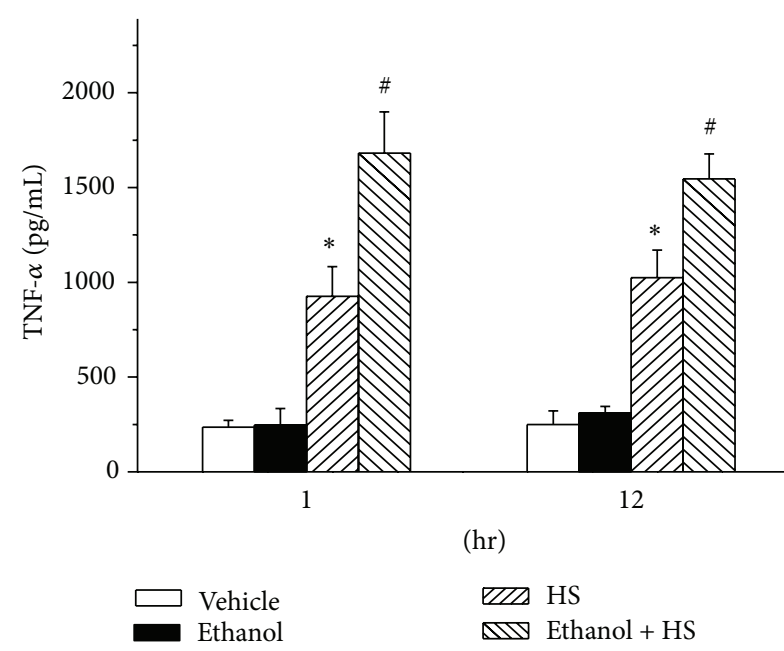

(a)

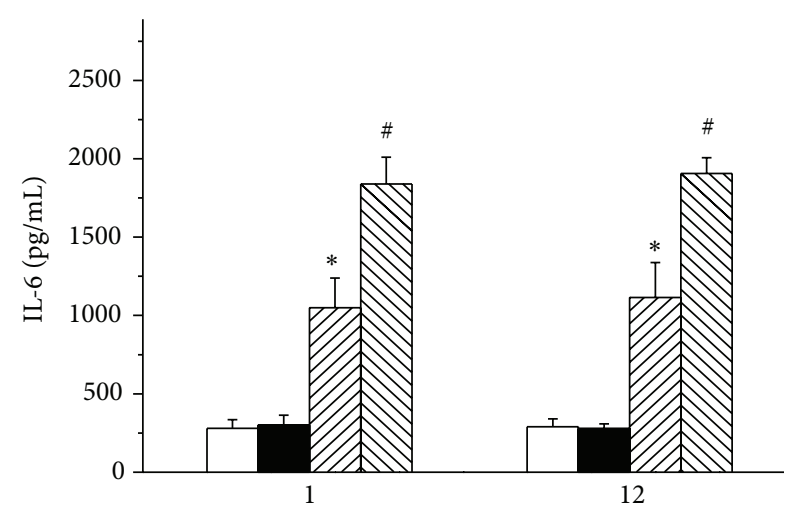

(hr)

EIA HS

लIV Ethanol + HS

(b)

FiguRE 6: Changes in serum (a) tumor necrosis factor- $\alpha$ (TNF- $\alpha$ ), and (b) interleukin-6 (IL-6) after hemorrhagic shock in rats. ${ }^{*} P<0.05$ for the HS group compared with the Vehicle group. ${ }^{\#} P<0.05$ for the Ethanol + HS group compared with the HS group.

usually related to blood alcohol concentration. At a blood alcohol concentration higher than $300 \mathrm{mg} / \mathrm{dL}$, there is an increased risk of respiratory depression and cardiac arrest. Death attributable to acute alcohol intoxication generally occurs at a blood alcohol concentration higher than $500 \mathrm{mg} / \mathrm{dL}$, although the lethal dose can vary [6]. In this study, blood alcohol concentration before HS was about $405.87 \pm 16.5 \mathrm{mg} / \mathrm{dL}-$ similar to concentrations observed in humans after binge drinking.

Alcohol intoxication aggravates traumatic injury-related hemodynamic instability [16]. Low MAP at the time of arrival into the emergency department has been reported to be a predictor of poor patient outcome from traumatic injury and blood loss [17]. Alcohol intoxication may impair the ability of blunt trauma patients to compensate for acute blood loss, making them more likely to be hypotensive on admission and increasing their need for packed red blood cells and intravenous fluids [18]. Our study noted heavy ethanol intoxication after hemorrhage had lower MAP and tachycardia than ethanol group. But there was no significant difference in MAP and HR between the HS group and the Ethanol + HS group in this study. We observed that after ethanol intoxication, hemoglobin increased first, which may be due to the higher osmolality of ethanol induced hemoconcentration. However, hemoglobin decreased at 18, 24 , and $48 \mathrm{~h}$ following hemorrhage in acute alcohol intoxicated rats compared with the HS only group. This might be because acute alcohol intoxication affected tissue hemorrhage 


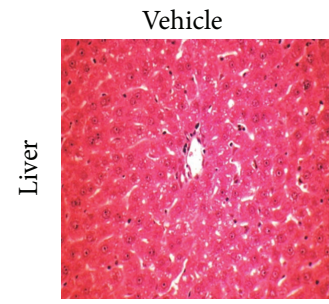

(a)

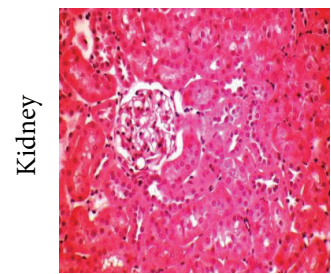

(b)

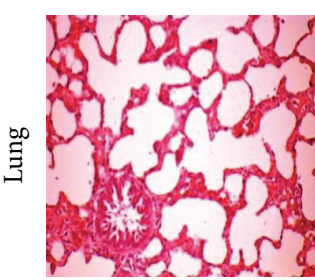

(c)

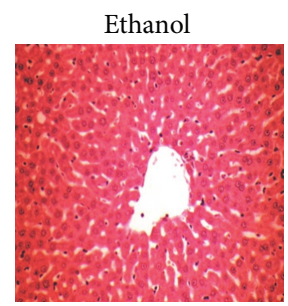

(d)

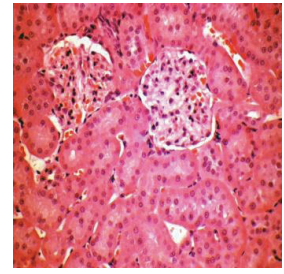

(e)

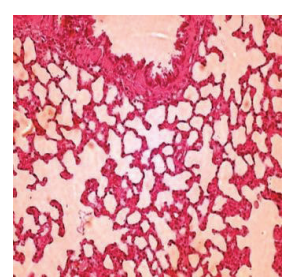

(f)

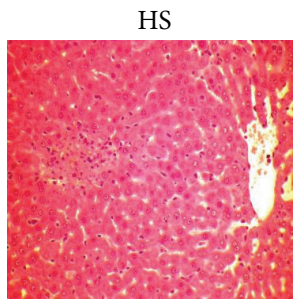

(g)

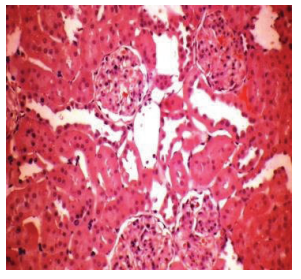

(h)

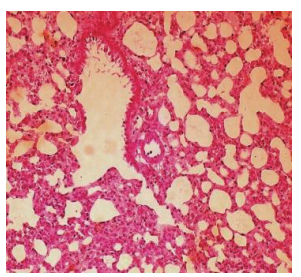

(i)

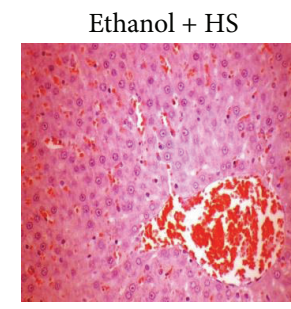

(j)

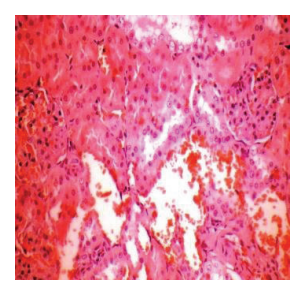

(k)

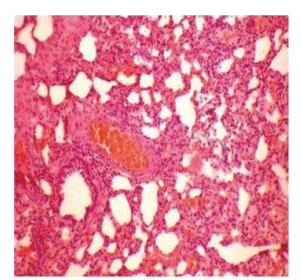

(1)

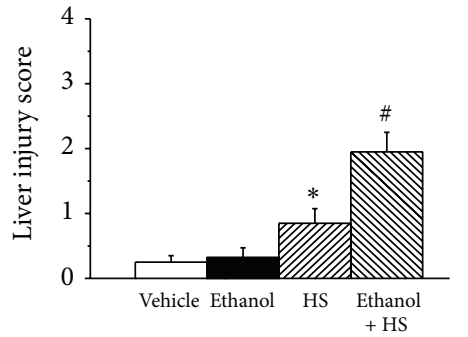

(m)

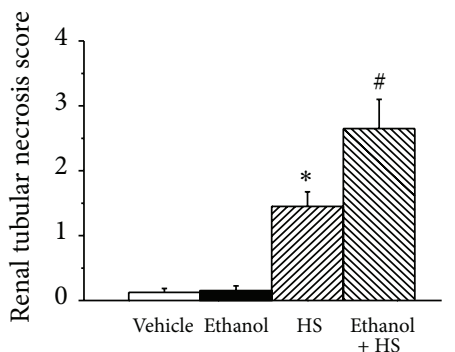

(n)

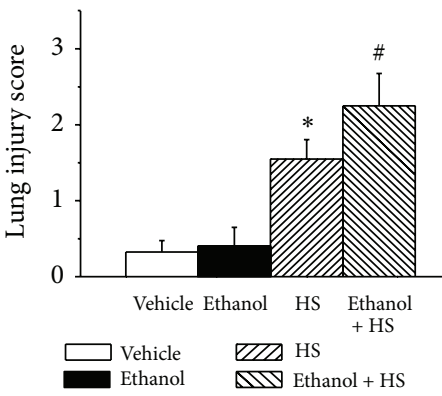

(o)

Figure 7: Histopathologic changes in the liver, kidneys, and lung after hemorrhagic shock in rats. Histologic sections from the Vehicle group ((a), (b), (c)), Ethanol group ((d), (e), and (f)), HS group ((g), (h), and (i)), and Ethanol + HS group ((j), (k), and (l)), stained with hematoxylin and eosin (liver, kidneys, lungs: magnification $\times 200$ ). Photomicrographs (a), (d), (g), and (j) are liver sections; (b), (e), (h), and (k) are kidney sections; (c), (f), (i), and (l) are lung sections. Histopathologic injury score in liver (m), kidney (n), and lung (o) after hemorrhagic shock in rats. ${ }^{*} P<0.05$ for the HS group compared with the Vehicle group. ${ }^{\sharp} P<0.05$ for the Ethanol + HS group compared with the HS group.

following HS. Pathology examination proved that the Ethanol + HS group had more tissue hemorrhage in the liver, kidney and lung.

The liver is particularly at risk for alcohol-related damage because it receives portal blood directly from the intestinal tract and thus experiences the highest concentration of alcohol presented to any organ [19]. In addition, ethanol metabolism in the liver produces potentially harmful toxic metabolites such as acetaldehyde, acetate, and reactive oxygen species [20]. Increased serum TNF- $\alpha$ and IL- 6 concentrations have frequently been found in alcoholic liver cirrhosis patients [21]. Alcohol intoxicated rodents, present with lower blood pressure at the time of injury, have decreased tolerance to blood loss and have impaired blood pressure recovery during fluid resuscitation. The accentuated hypotension leads to tissue hypoperfusion, which enhances susceptibility to tissue injury reflected in greater elevation in circulating liver function [22]. Our study also observed that acute ethanol intoxication aggravated liver damage by greater elevation of GOT, GPT, and the histopathologic analysis of liver revealed more hepatocyte necrosis and leukocytes infiltration following hemorrhage in acute alcohol intoxicated rats.

Alcohol consumption increased malondialdehyde levels, superoxide dismutase, and catalase activity significantly in alcohol intoxicated rats [23]. Alcohol intoxicated rats result in a greater reduction of blood flow to the kidney than that seen in nonintoxicated rats after HS [24]. The accentuated hypotension leads to tissue hypoperfusion, which enhances susceptibility to tissue injury reflected in greater elevation in circulating renal function [23]. Our study found that acute ethanol intoxication induced greater renal damage after HS in rats by elevated serum BUN, Cre and exacerbated the histopathologic changes in kidneys following HS in rats.

Alcohol exposure of the host can predispose to pneumonia infection [9]. Acute alcohol intoxication exacerbates the HS-induced increase in lung proinflammatory cytokine TNF- $\alpha$ expression in rats [25]. In other studies, alcoholtreated mice had worse clinical outcomes, deteriorated pulmonary structure, and increased levels of IL-6 compared with the nonalcohol treated mice [26]. Our study found 
that heavy ethanol intoxication aggravated lung damage including pulmonary edema, hemorrhage, and interstitial PMN inflammatory cell infiltration in the lung following hemorrhage.

In response to $\mathrm{HS}, \mathrm{NF}-\kappa \mathrm{B}$ is involved in apoptosis and the inflammatory cascade [5]. The organism provokes release of proinflammatory cytokines (TNF- $\alpha$ and IL-6) into surrounding tissues, thereby causing tissue damage and organ failure $[2,3]$. TNF- $\alpha$ and IL- 6 peak early after HS and continue elevating during $\mathrm{HS}$ because tissue hypoperfusion persist [10-12]. Treatment with anti- TNF- $\alpha$ antibodies reduced organ injury and improved survival in rats after HS [27]. Inhibition of the synthesis of IL- 6 may exert beneficial effects on HS [28]. Acute alcohol intoxication can increase proinflammatory cytokine production and induce marked immunosuppression after HS [6]. Our study found that intravenous injection of heavy ethanol increased serum TNF$\alpha$ and IL- 6 production after HS in rats. Altered inflammatory cell and adaptive immune responses after alcohol consumption result in increased of infections and other organ-specific immune-mediated effects [9].

Sex differences in alcohol drinking is somewhat equivocal in rodent studies: one study noted female rodents tend to drink more alcohol than males [29] and other study noted adolescent males have been reported to drink more than females [30], whereas others suggest that there is no sex difference [31, 32]. Increases in pubertal hormones, including gonadal and stress hormones, are a prominent developmental feature of adolescence and could contribute to the progression of sex differences in alcohol drinking patterns during puberty [33]. Our study used adult male Wistar-Kyoto rats and found increased serum TNF- $\alpha$ and IL-6 levels after HS and aggravated HS-induced organ damage. Further studies are required to investigate sex differences in alcohol drinking behavior and/or the influence of pubertal hormone changes on the effects of ethanol on physiopathology and cytokine levels following HS in acutely alcohol-intoxicated rats.

Recent study noted that rats were given a single oral dose of ethanol $(5 \mathrm{~g} / \mathrm{kg}, 30 \%)$ increased survival after HS and decreased HS-induced liver injury [34]. However, the blood ethanol concentration in this study is unknown and only given a single oral dose of ethanol. Our study, blood alcohol concentration before HS was similar to concentrations observed in humans after binge drinking. Acute ethanol intoxication leads to a dysregulation of the hemodynamic, neuroendocrine, inflammatory, and immune responses to hemorrhage. This disruption of the normal neuroendocrine counterregulatory response impairs hemodynamic stability and recovery, contributing to compromised tissue perfusion and increased end-organ injury $[6,22]$. Intravenous heavy ethanol injection of this study also noted increased serum TNF- $\alpha$ and IL- 6 levels after HS and aggravated HS-induced organ damage in rats.

\section{Conclusion}

Acute ethanol intoxication increased serum TNF- $\alpha$ and IL6 levels following HS, along with aggravating HS-induced organ damage in rats.

\section{Conflict of Interests}

The authors report no conflict of interests related to this study.

\section{Acknowledgments}

This work was supported in part by grants from the Tzu Chi University (TCIRP 98004-02) and National Science Council (NSC100-2314-B-303-013).

\section{References}

[1] N. Curry, S. Hopewell, C. Dorée, C. Hyde, K. Brohi, and S. Stanworth, "The acute management of trauma hemorrhage: a systematic review of randomized controlled trials," Critical Care, vol. 15, no. 2, article R92, 2011.

[2] M. Keel and O. Trentz, "Pathophysiology of polytrauma," Injury, vol. 36, no. 6, pp. 691-709, 2005.

[3] M. K. Angele, C. P. Schneider, and I. H. Chaudry, "Bench-tobedside review: latest results in hemorrhagic shock," Critical Care, vol. 12, no. 4, article 218, 2008.

[4] M. Frink, Y. Hsieh, B. M. Thobe et al., "TLR4 regulates Kupffer cell chemokine production, systemic inflammation and lung neutrophil infiltration following trauma-hemorrhage," Molecular Immunology, vol. 44, no. 10, pp. 2625-2630, 2007.

[5] G. D. Rushing and L. D. Britt, "Reperfusion injury after hemorrhage: a collective review," Annals of Surgery, vol. 247, no. 6, pp. 929-937, 2008.

[6] P. Greiffenstein and P. E. Molina, "Alcohol-induced alterations on host defense after traumatic injury," Journal of Trauma, vol. 64, no. 1, pp. 230-240, 2008.

[7] P. E. Molina, J. K. Sulzer, and A. M. Whitaker, "Alcohol abuse and the injured host: dysregulation of counterregulatory mechanisms review," Shock, vol. 39, pp. 240-249, 2013.

[8] L. Vonghia, L. Leggio, A. Ferrulli, M. Bertini, G. Gasbarrini, and G. Addolorato, "Acute alcohol intoxication," European Journal of Internal Medicine, vol. 19, no. 8, pp. 561-567, 2008.

[9] G. Szabo and P. Mandrekar, "A recent perspective on alcohol, immunity, and host defense," Alcoholism, vol. 33, no. 2, pp. 220$232,2009$.

[10] C. Lee, R. Lee, Y. Subeq, C. Lee, T. Chen, and B. Hsu, "Fluvastatin attenuates severe hemorrhagic shock-induced organ damage in rats," Resuscitation, vol. 80, no. 3, pp. 372-378, 2009.

[11] W. Wu, N. Lin, Y. Subeq, R. Lee, I. Chen, and B. Hsu, "Erythropoietin protects severe haemorrhagic shock-induced organ damage in conscious rats," Injury, vol. 41, no. 7, pp. 724$730,2010$.

[12] F. Yang, Y. Subeq, C. Lee, R. Lee, T. Peng, and B. Hsu, "Melatonin ameliorates hemorrhagic shock-induced organ damage in rats," Journal of Surgical Research, vol. 167, no. 2, pp. e315-e321, 2011.

[13] J. Werner, M. Saghir, A. L. Warshaw et al., "Alcoholic pancreatitis in rats: Injury from nonoxidative metabolites of ethanol," American Journal of Physiology, vol. 283, no. 1, pp. G65-G73, 2002.

[14] H. B. Lee and M. D. Blaufox, "Blood volume in the rat," Journal of Nuclear Medicine, vol. 26, no. 1, pp. 72-76, 1985.

[15] J. B. A. MacLeod and D. W. Hungerford, "Alcohol-related injury visits: do we know the true prevalence in U.S. trauma centres?" Injury, vol. 42, no. 9, pp. 922-926, 2011. 
[16] H. Shih, S. Hu, C. Yang, T. Ko, J. Wu, and C. Lee, "Alcohol intoxication increases morbidity in drivers involved in motor vehicle accidents," American Journal of Emergency Medicine, vol. 21, no. 2, pp. 91-94, 2003.

[17] S. R. Heckbert, N. B. Vedder, W. Hoffman et al., "Outcome after hemorrhagic shock in trauma patients," The Journal of trauma, vol. 45, no. 3, pp. 545-549, 1998.

[18] J. Bilello, V. McCray, J. Davis, L. Jackson, and L. A. Danos, "Acute ethanol intoxication and the trauma patient: hemodynamic pitfalls," World Journal of Surgery, vol. 35, no. 9, pp. 2149-2153, 2011.

[19] A. M. Karinch, J. H. Martin, and T. C. Vary, "Acute and chronic ethanol consumption differentially impact pathways limiting hepatic protein synthesis," American Journal of Physiology, vol. 295, no. 1, pp. E3-E9, 2008.

[20] C. S. Lieber, "Ethanol metabolism, cirrhosis and alcoholism," Clinica Chimica Acta, vol. 257, no. 1, pp. 59-84, 1997.

[21] J. Daniluk, A. Szuster-Ciesielska, J. Drabko, and M. KandeferSzerszeń, "Serum cytokine levels in alcohol-related liver cirrhosis," Alcohol, vol. 23, no. 1, pp. 29-34, 2001.

[22] P. E. Molina, J. K. Sulzer, and A. M. Whitaker, "Alcohol abuse and the injured host: dysregulation of counterregulatory mechanisms review," Shock, vol. 39, pp. 240-249, 2013.

[23] M. F. Sönmez, F. Narin, D. Akkuş, and A. B. Türkmen, "Melatonin and vitamin $\mathrm{C}$ ameliorate alcohol-induced oxidative stress and eNOS expression in rat kidney," Renal Failure, vol. 34, no. 4, pp. 480-486, 2012.

[24] J. K. Sulzer, A. M. Whitaker, and P. E. Molina, "Hypertonic saline resuscitation enhances blood pressure recovery and decreases organ injury following hemorrhage in acute alcohol intoxicated rodents," The Journal of Trauma and Acute Care Surgery, vol. 74, pp. 196-202, 2013.

[25] P. E. Molina, K. L. Zambell, K. Norenberg et al., "Consequences of alcohol-induced early dysregulation of responses to trauma/hemorrhage," Alcohol, vol. 33, no. 3, pp. 217-227, 2004.

[26] C. D. Spies, N. Lanzke, U. Schlichting et al., "Effects of ethanol on cytokine production after surgery in a murine model of gram-negative pneumonia," Alcoholism, vol. 32, no. 2, pp. 331338, 2008.

[27] S. Bahrami, Y. Yao, G. Leichtfried, H. Redl, I. Marzi, and G. Schlag, "Significance of TNF in hemorrhage-related hemodynamic alterations, organ injury, and mortality in rats," American Journal of Physiology, vol. 272, no. 5, pp. H2219-H2226, 1997.

[28] S. I. Brundage, N. A. Zautke, J. B. Holcomb et al., "Interleukin-6 infusion blunts proinflammatory cytokine production without causing systematic toxicity in a swine model of uncontrolled hemorrhagic shock," Journal of Trauma, vol. 57, no. 5, pp. 970978, 2004.

[29] F. E. Lancaster and K. S. Spiegel, "Sex differences in pattern of drinking," Alcohol, vol. 9, no. 5, pp. 415-420, 1992.

[30] C. Vetter-O'Hagen, E. Varlinskaya, and L. Spear, "Sex differences in ethanol intake and sensitivity to aversive effects during adolescence and adulthood," Alcohol and Alcoholism, vol. 44, no. 6, pp. 547-554, 2009.

[31] E. M. Truxell, J. C. Molina, and N. E. Spear, "Ethanol intake in the juvenile, adolescent, and adult rat: effects of age and prior exposure to ethanol," Alcoholism: Clinical and Experimental Research, vol. 31, no. 5, pp. 755-765, 2007.

[32] M. Morales, E. I. Varlinskaya, and L. P. Spear, "Age differences in the expression of acute and chronic tolerance to ethanol in male and female rats," Alcoholism, vol. 35, no. 9, pp. 1614-1624, 2011.
[33] E. D. Witt, "Puberty, hormones, and sex differences in alcohol abuse and dependence," Neurotoxicology and Teratology, vol. 29, no. 1, pp. 81-95, 2007.

[34] B. Relja, C. Höhn, F. Bormann et al., "Acute alcohol intoxication reduces mortality, inflammatory responses and hepatic injury after haemorrhage and resuscitation in vivo," British Journal of Pharmacology, vol. 165, no. 4 B, pp. 1188-1199, 2012. 


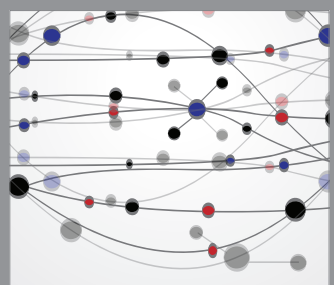

The Scientific World Journal
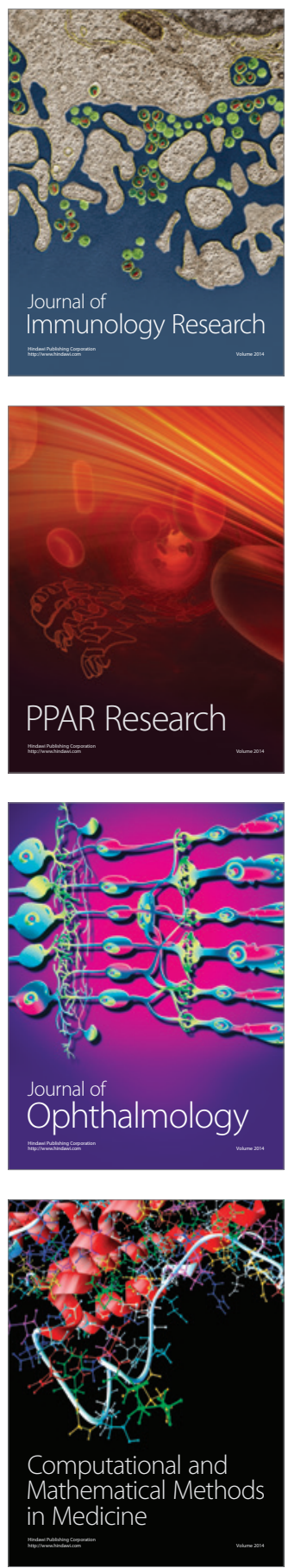

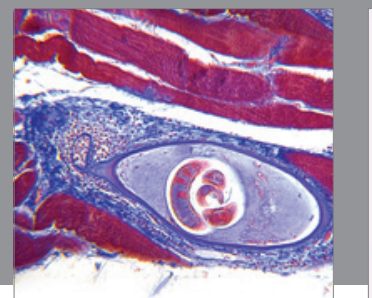

Gastroenterology

Research and Practice
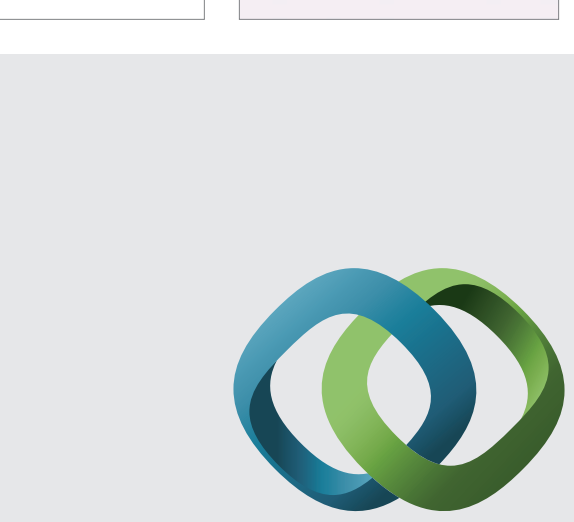

\section{Hindawi}

Submit your manuscripts at

http://www.hindawi.com
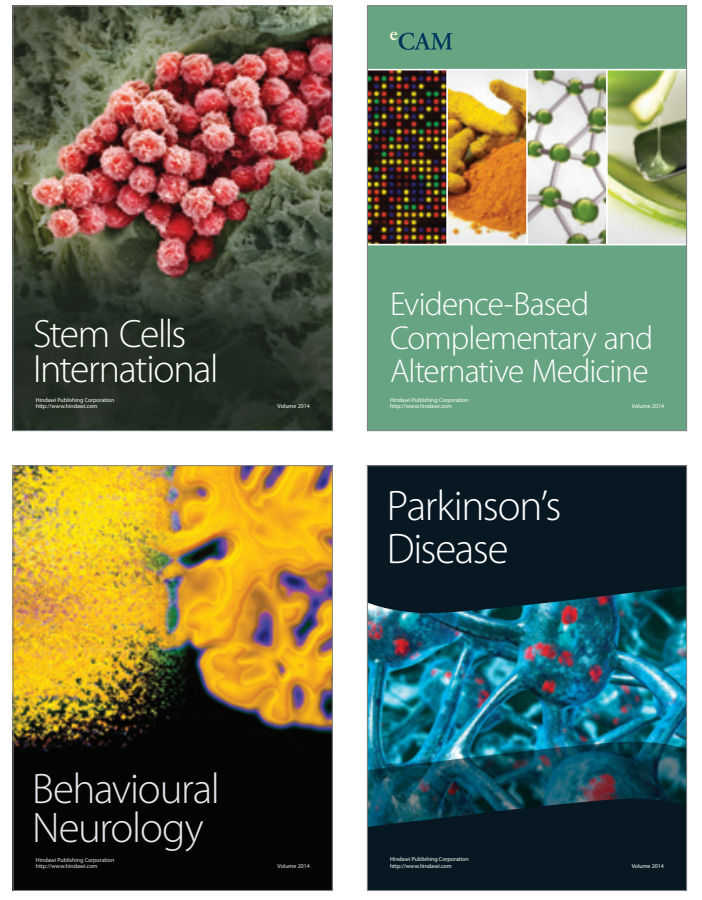
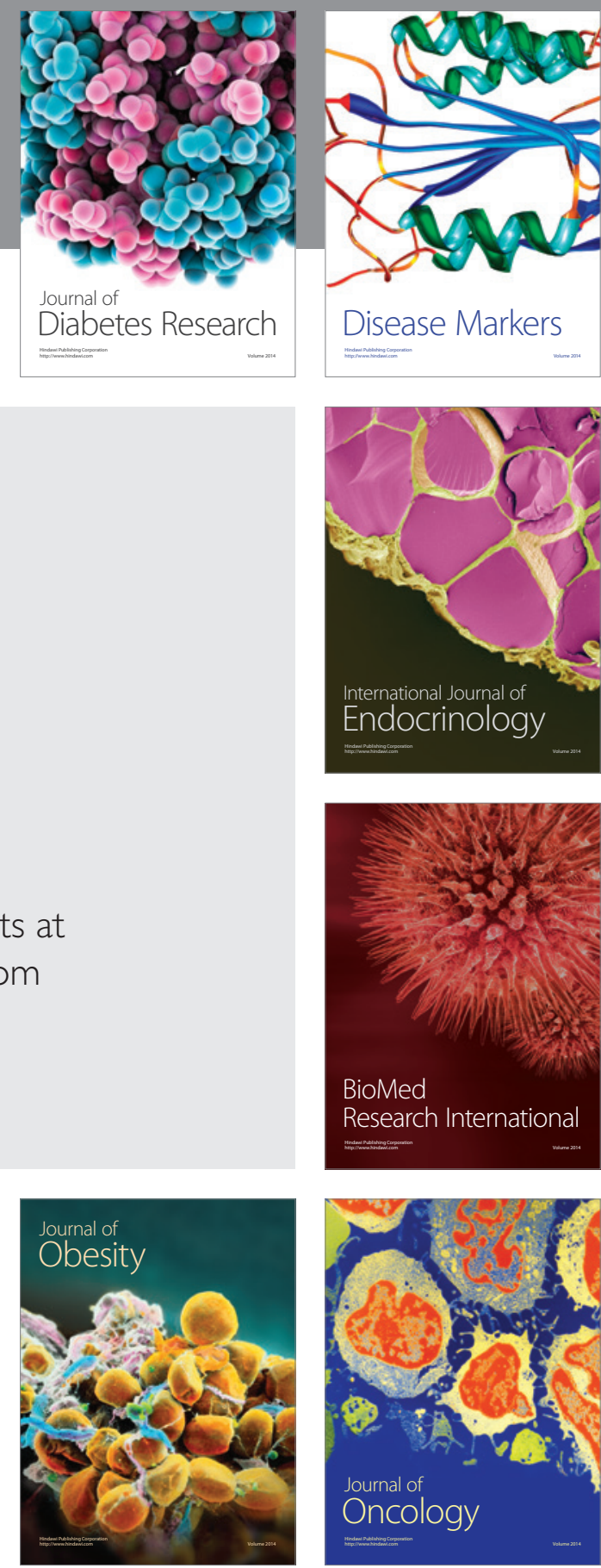

Disease Markers
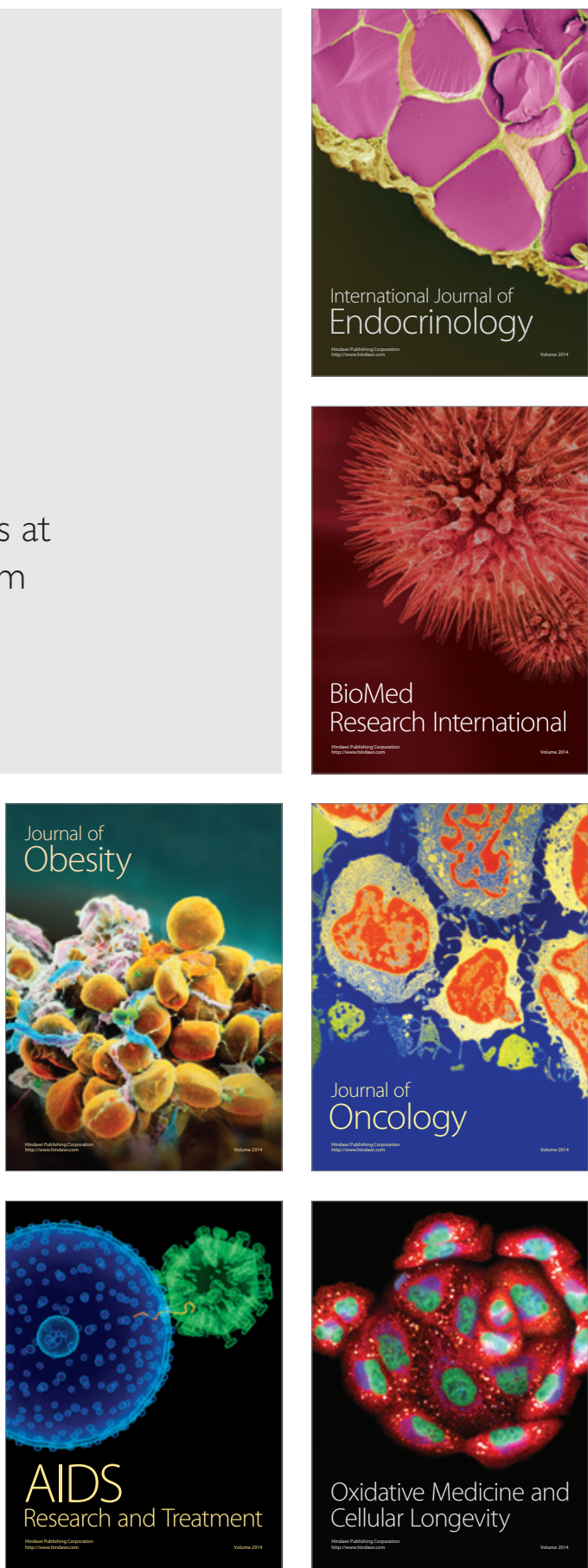